chemotherapy significantly improved overall survival rates, without recurrence, and reduced the rate of distant metastatic dissemination. The objective of this work is to describe the histological response of cervical cancer treated with concomitant radiotherapy and chemotherapy (CCRT) followed by surgery, as well as the preoperative difficulties and morbidity related to surgery

Methodology This is a retrospective study of 126 patients treated for cervical cancer by CCRT followed by surgery at the Med VI Center for Gyneacologic and Breast Cancer Treatment at the UHC Ibn Rochd from January 2016 to December 2018.

Results The average age of the patients was 51, the mean total time from symptom onset to medical consultation was 7,5 months. Stage IIB was the discovery stage in $71 \%$ of the patients. Cervical biopsy results showed squamous cell carcinoma in 79\%, adenocarcinoma in $16 \%$ and $5 \%$ of patients had other histological types.

All of our patients received a weekly chemotherapy of $40 \mathrm{mg}$ of cisplatin, 4 cycles on average, associated with external radiotherapy sessions reaching 45 and 50 Gy supplemented by brachytherapy for 68 patients, $46 \%$ of patients were referred for surgery without additional brachytherapy most often due to lack of means. Surgical treatment, radical hysterectomy with salpingo-oophorectomy and bilateral pelvic lymphadenectomy was performed in $91.26 \%$ and $75.55 \%$ had a radical hysterectomy due to peroperative difficulties. The tumor residue was macroscopic in 29 patients. The surgical margins were positive in 8 cases. Parameters were invaded in 5 patients, 22 cases showed positive vascular emboles, Lymph node curage was positive in 14 cases.

Conclusion The overall treatment period is a main prognostic factor and second surgery following CCRT remains a great concern because of its morbidity.

Disclosures The authors declare they have no conflict of interest

\section{PREOPERATIVE BRACHYTHERAPY FOLLOWED BY LAPAROSCOPIC HYSTERECTOMY: A NEW OPTION TO CONSIDER FOR EARLY STAGES CERVICAL CANCER IN THE LIGHT OF THE LACC TRIAL RESULTS}

\footnotetext{
${ }^{1}$ Clemence Beyer, ${ }^{2}$ Houssein EL Hajj, ${ }^{3}$ Laurence Gonzague, ${ }^{3}$ Leonel Varela, ${ }^{3}$ Camille Jauffret, ${ }^{3}$ Guillaume Blache, ${ }^{3}$ Laura Sabiani, ${ }^{3}$ Gilles Houvenaeghel, ${ }^{3}$ Magalie Provansal, ${ }^{3}$ Renaud Sabatier, ${ }^{3}$ Eric Lambaudie. ${ }^{1}$ Grenoble Alpes University Hospital Center; ${ }^{2}$ Centre Léon Bérard; ${ }^{3}$ Paoli Calmettes Institute
}

\subsection{6/ijgc-2020-ESG0.44}

Objectives The aim of this study is to report the oncological and surgical outcomes of an alternative treatment strategy to upfront surgery for patients presenting with early stage cervical cancer (ESCC) (FIGO 2018 stages IA1-IB2). This treatment strategy consists of a combination preoperative brachytherapy (POBT) followed by a Querleu Morrow Type A laparoscopic or abdominal hysterectomy.

Material and Methods This retrospective study was conducted at the Marseille regional tertiary cancer center in France for patients treated for ESCC (FIGO 2018 stages IA1-IB2) between 2001 to 2012. All patients underwent a Low Dose Rate (LDR) POBT after confirming the absence of pelvic lymph node metastasis (radiological and surgical staging). 6 to 8 weeks after brachytherapy completion, all patients underwent a Querleu Morrow Type A hysterectomy (laparotomy and minimally invasive). The primary endpoint was the Disease Free Survival (DFS) and the secondary endpoint was the morbidity related to this radio-surgical multimodal approach.

Results A total of 138 patients were included. Histological analysis showed a complete response in 68 patients $(49.3 \%)$ and a residual tumor $<1 \mathrm{~cm}$ in 36 patients (26\%).

With a median follow up of 132 months (60 - 204 months), DFS was $93.5 \%$ and 9 recurrences occurred (1 local pelvic recurrence, 2 pelvic lymph node recurrences and 6 distant recurrences).

In univariate analysis, we found that a duration between the completion of brachytherapy and surgery exceeding 52 days is associated with a significant decrease in DFS ( $\mathrm{p}=$ 0.004$, OR $=8.5,95 \%$ CI $\{1.5 ; 48.7\})$. Pathological complete response was found to be associated with an increased DFS (p $=0.03 \mathrm{OR}=6.195 \% \mathrm{CI}\{1.8 ; 55.3\}$ ).

The brachytherapy related rate of late complications was $17.3 \% \quad(n=24) \quad$ (Chassagne glossary) and the surgery related urinary tract complications rate was $6.5 \%(n=9)$, with only 2 patients $(1.5 \%)$ presented grade 3 complications (Clavien Dindo classification).

Conclusion After a median follow up of 132 months, the multimodal radio-surgical management of ESCC (FIGO 2018 Stages IA1-IB2) consisting of POBT followed by a Querleu Morrow Type A laparoscopic hysterectomy appears to be a reasonable alternative to upfront open radical hysterectomy particularly in patients with high risk ESCC $(<2 \mathrm{~cm}$ associated with negative prognostic factors or for tumors measuring between 2 and $4 \mathrm{~cm}$ ).

This multimodal radio-surgical approach is associated with a low rate of complications and a reasonable rate of local recurrences compared to the results of the LACC trial. Further studies are necessary to confirm these results.

Disclosures Doctors Clémence Beyer, Houssein El Hajj, Laurence Gonzague, Leonel Varela Cagetti, Camille Jauffret-Fara, Guillaume Blache, Laura Sabiani, Magalie Provansal and Renaud Sabatier have no conflicts of interest or financial ties to disclose.

Gilles Houvenaeghel and Eric Lambaudie are proctors for Intuitive Surgical.

\section{ROBOTIC RADICAL PARAMETRECTOMY IN PATIENTS WITH UNDIAGNOSED INVASIVE CERVICAL CANCER: A STEP BY STEP PROCEDURE}

${ }^{1}$ Houssein EL Hajj, ${ }^{2}$ Domenico Ferraioli, ${ }^{2}$ Mathilde Roussel, ${ }^{2}$ Jauffret Camille, ${ }^{2}$ Gilles Houvenaeghel, ${ }^{2}$ Eric Lambaudie. ${ }^{1}$ Centre Léon Bérard; ${ }^{2}$ Paoli Calmettes Institute

\subsection{6/ijgc-2020-ESGO.45}

Introduction/Background Occult discovery of invasive cervical cancer discovered after hysterectomy for non-malignant tumors is not uncommon. For patients presenting an incidental diagnosis of early stage invasive cervical cancer (FIGO Stages IA1IB2), two possible strategies can be proposed: Adjuvant radiation Therapy with no tumor target or Radical Parametrectomy (RP) associated with upper vaginectomy and pelvic lymph node dissection.

Methodology The procedure starts by incising the peritoneum on the lateral pelvic sidewall. Dissection is proceeded from the round ligament stump towards the ligated 
infundibulo-pelvic pedicle stump, followed by the development of the retroperitoneal space with the identification of the umbilical artery, the iliac vessels and laterally the psoas muscle with the genito-femoral nerve. Paravesical and pararectal spaces are developed down to the pelvic floor. A radical pelvic lymphadenectomy is performed bilaterally. Once lymph node involvement is excluded, we proceed to parametrectomy.

Radical parametrectomy is started with the dissection from the posterior leaf of the broad ligament. The anterior division of the internal iliac artery (IIA) is identified and the uterine artery and vein are transected proximally at their origin using a vascular sealing system or Hem-O-Lok clips. The ureters are then mobilized from their attachments and separated from the medial leaf of the peritoneum down to the ureteral tunnel below the uterine artery and to their entrance into the bladder. Aided by the vaginal probe, the bladder peritoneum is incised, and the bladder is dissected and mobilized inferiorly down to the middle third of the vagina. After dissection of the bladder pillar, the vesico-vaginal space is joined to the paravesical space, completely separating the bladder from the anterior vaginal wall. In cases of anatomical distortion or bladder adhesions, instillation of $300 \mathrm{cc}$ of saline solution associated with Methylene blue dye in the bladder might be required to guide the dissection. Posteriorly, the peritoneum is incised at the level of the cul-de-sac of Douglas and the rectovaginal space is developed isolating the uterosacral ligaments. The proximal parametrium and para-vaginal tissues are finally dissected as in a Type B1 Querleu Morrow radical hysterectomy.

The same procedure is performed on both sides. A circular incision is made about $3 \mathrm{~cm}$ below the vaginal cuff aided by upward vaginal traction.

Results When compared to Radiation therapy RP presents a lower rate of late complications, making it the preferred approach to treat younger patients. Traditionally performed via laparotomy, minimally invasive approach is now proven feasible and effective.

Conclusion This article presents a focused anatomic review and describes the surgical technique of the five-port robotic assisted radical parametrectomy.

Disclosures Eric Lambaudie and Gilles Houvenaeghle report grants and personal fees from Intuitive Surgical, outside the submitted work.

The other authors have no other conflicts of interest to declare.

\section{ENHANCED RECOVERY AFTER SURGERY (ERAS) FOR PARA-AORTIC LYMPHADENECTOMY-A NEW TREND TO CONSIDER?}

${ }^{1}$ Isabelle Masquin, ${ }^{2}$ Houssein EL Hajj, ${ }^{1}$ Christophe Zemour, ${ }^{1}$ Camille Jauffret Farra, ${ }^{1}$ Guillaume Blache, ${ }^{1}$ Mellie Heinemann, 'Laura Sabiani, ${ }^{1}$ Clement Brun, ${ }^{1}$ Gilles Houvenaeghel, ${ }^{1}$ Marion Faucher, ${ }^{1}$ Eric Lambaudie. ${ }^{1}$ Paoli Calmettes Institute; ${ }^{2}$ Centre Léon Bérard

\subsection{6/ijgc-2020-ESGO.46}

Background Enhanced recovery after surgery (ERAS) is a multimodal approach aiming to improve rehabilitation after surgery. In gynecologic malignancies, para-aortic lymphadenectomy (PAL) is indicated for either diagnostic or therapeutic finalities. Minimally invasive surgery (MIS) constitutes the cornerstone for ERAS programs.

Methods This retrospective study conducted between November 2006 and January 2018, aims to analyze the role of ERAS implementation for patients undergoing PAL. Starting 2016, an ERAS protocol was implemented for all the patients in our institution. All patients who underwent PAL for gynecologic malignancies were included in this study. To analyze the impact of this implementation on the surgical outcomes (length of hospital stay (LOS)) and the post-operative complications, we compared the patients who underwent PAL within ERAS protocol between 2016 and 2018 'ERAS Group' to the patients who underwent PAL prior to this implementation (between 2006 and 2015) 'Prior to ERAS group'.

Results A total of 193 patients were identified. 'ERAS Group' was associated with a significant decrease of median LOS (2 days vs. 3 days, $\mathrm{p}<0.001)$ and a significant increase in earlier post-operative discharges: $\mathrm{OR}=29.62$ [13.58-64.64], $\mathrm{p}<0.001$. Two factors were independently associated with early postoperative discharge: Implementation of the ERAS protocol $(\mathrm{OR}=25.64$ [8.14-80.71], $\mathrm{p}<0.0001)$ and the endorsement of the extraperitoneal technique for $\mathrm{PAL}(\mathrm{OR}=5.92 \quad[2.10$ 16.68], $p=0.0008)$. There was no difference in intra-operative complications rate between groups $(\mathrm{p}=0.497)$. More postoperative complications were found in the 'ERAS group' $(23 \%$ vs $10 \%, \mathrm{p}=0.017$ ) but this difference was not significant for severe complications $(\mathrm{p}=0.277)$ and lymphocele rate $(\mathrm{p}=0.248)$.

Conclusions Implementing ERAS protocols for patients undergoing minimally invasive PAL is an independent factor improving early recovery and decreasing the LOS without increasing severe complications.

Disclosures Drs Isabelle Masquin, Mellie Heinemann, Christophe Zemmour, Camille Jauffret-Fara, Guillaume Blache, Laura Sabiani, Clément Brun, Marion Faucher and Houssein El Hajj have no conflicts of interest or financial ties to disclose. Gilles Houvenaeghel and Eric Lambaudie are proctors for Intuitive Surgical.

\section{Diagnostics}

\section{A NEW PROPOSAL FOR THE CLINICAL CLASSIFICATION OF VULVAR LICHEN SCLEROSUS: AN OBSERVATIONAL PROSPECTIVE STUDY}

${ }^{1}$ Veronica Boero, ${ }^{2}$ Carlo A Liverani, ${ }^{3}$ Massimiliano Brambilla, ${ }^{1}$ Ermelinda Monti ${ }^{4}$ Filippo Murina, ${ }^{5}$ Roberto Tozzi, ${ }^{6}$ Eugenia DI Loreto, ${ }^{1}$ Chiara Maria F Dell'utri, ${ }^{1}$ Giada Libutti, ${ }^{7}$ Fabio Parazzini. ${ }^{1}$ Fondazione Irccs Ca' Granda - Ospedale Maggiore Policlinico; Gynecologic Unit; ${ }^{2}$ Humanitas San Pio X; Department of Gynecology; ${ }^{3}$ Fondazione Irccs Ca' Granda - Ospedale Maggiore Policlinico; Plastic Surgery Service, Gynecology Unit; ${ }^{4}$ Ospedale Vittore Buzzi; Lower Genital Tract Disease Unit, Obstetrics and Gynecology Department; ${ }^{5}$ Women's Centre (Level 3), John Raddliffe Hospital; University of Oxford; Department of Gynaecologic Oncology, Nuffield Department of Women's and Reproductive Health (Wrh); ${ }^{6}$ Fondazione Irccs Ca' Granda - Ospedale Maggiore Policlinico; Department of Gynecology; ${ }^{7}$ University Hospital of Milan; Department of Clinical Sciences and Community Health

\subsection{6/ijgc-2020-ESG0.47}

Introduction/Background Vulvar Lichen Sclerosus (VLS) is a chronic inflammatory disorder which commonly affects the female anogenital epithelium, leading to scarring, anatomical 